\title{
Current state of perinatal postmortem magnetic resonance imaging: European Society of Paediatric Radiology questionnaire-based survey and recommendations
}

\author{
Elspeth Whitby ${ }^{1}$ (D) Amaka C. Offiah ${ }^{2,3} \cdot$ Susan C. Shelmerdine ${ }^{4,5} \cdot$ Rick R. van Rijn $^{6} \cdot$ Michael Aertsen $^{7}$. \\ Willemijn M. Klein ${ }^{8}$ - David Perry ${ }^{9}$ - Stacy K. Goergen ${ }^{10,11} \cdot$ Christian Abel $^{12}$ - Ajay Taranath $^{13,14} \cdot$ Dominic Gascho $^{15}$. \\ Elka Miller $^{16}$. Owen J. Arthurs ${ }^{4,5}$
}

Received: 13 May 2020 / Revised: 2 September 2020 / Accepted: 9 November 2020 / Published online: 23 December 2020

(C) The Author(s) 2020

\begin{abstract}
Background Postmortem magnetic resonance imaging (MRI) in perinatal and childhood deaths is increasingly used as a noninvasive adjunct or alternative to autopsy. Imaging protocols vary between centres and consensus guidelines do not exist.

Objective Our aim was to develop practical, standardised recommendations for perinatal postmortem MRI.

Materials and methods Recommendations were based on the results of two surveys regarding local postmortem MRI practices sent electronically to all 14 members of the European Society of Paediatric Radiology (ESPR) Postmortem Imaging Task Force and 17 members of the International Society of Forensic Radiology and Imaging Task Force (25 different centres).

Results Overall, 11/14 (78.6\%) respondents from different institutions perform postmortem MRI. All of these centres perform postmortem MRI for perinatal and neonatal deaths, but only 6/11 (54.5\%) perform imaging in older children.

Conclusion We propose a clinical standard for postmortem MRI sequences plus optional sequences for neuroimaging and cardiac anatomy depending on available scanning time and referral indications.
\end{abstract}

Keywords Consensus recommendations $\cdot$ Foetal $\cdot$ Imaging $\cdot$ Magnetic resonance imaging $\cdot$ Perinatal $\cdot$ Postmortem $\cdot$ Protocol $\cdot$ Survey

Elspeth Whitby

e.whitby@sheffield.ac.uk

1 University of Sheffield and Sheffield Teaching Hospitals Foundation Trust, Jessop Wing, Tree Root Walk, Sheffield S10 1SF, UK

2 Academic Unit of Child Health, University of Sheffield, Sheffield, UK

3 Department of Radiology, Sheffield Children's NHS Foundation Trust, University of Sheffield, Sheffield, UK

4 Department of Clinical Radiology, Great Ormond Street Hospital for Children, London, UK

5 UCL Great Ormond Street Institute of Child Health, London, UK

6 Department of Radiology and Nuclear Medicine, Amsterdam UMC, University of Amsterdam, Amsterdam, The Netherlands

7 Department of Radiology, University Hospitals KU Leuven, Leuven, Belgium
8 Department of Radiology and Nuclear Medicine and Anatomy, Radboud University Medical Centre, Nijmegen, the Netherlands

9 Radiology Department, National Women's Health and Starship Children's Hospital, Auckland City Hospital, Auckland, New Zealand

10 Monash Imaging, Clayton, Victoria, Australia

11 School of Clinical Sciences, Monash University, Clayton, Victoria, Australia

12 Department of Medical Imaging, John Hunter Hospital, Newcastle, New South Wales, Australia

13 Department of Medical Imaging, Women's and Children's Hospital, North Adelaide, South Australia, Australia

14 University of Adelaide, Adelaide, South Australia, Australia

15 Zurich Institute of Forensic Medicine, University of Zurich, Zurich, Switzerland

16 Department of Medical Imaging, Children's Hospital of Eastern Ontario, University of Ottawa, Ottawa, Canada 


\section{Introduction}

Paediatric postmortem magnetic resonance imaging (MRI) was first reported in 1996 by Brookes et al. [1]. Since then, there have been numerous studies by research groups worldwide [2-4] assessing the value of paediatric postmortem MRI in addition to or instead of autopsy. Initially, the central nervous system was assessed by paediatric postmortem MRI with high diagnostic accuracy [5]; more recently, whole-body imaging including paediatric postmortem MRI has been used to predict organ weights [6] and guide biopsy [7, 8]. Researchers have also investigated higher field MRI compared to conventional MRI [9] to evaluate small structures such as the heart [10]. The largest study to date of 400 cases showed high diagnostic accuracy in foetuses and stillbirths [11], particularly for the brain, abdomen and heart [12-14].

While several centres now use paediatric postmortem MRI in routine clinical practice, there are no consensus guidelines for paediatric/perinatal postmortem MRI protocols. The European Society of Paediatric Radiology (ESPR) set up a task force in 2016 to establish the extent and nature of current clinical utilisation and to develop minimum standards for clinical radiology practice. In this manuscript, we outline a questionnaire-based survey of postmortem MRI protocols used by ESPR and International Society of Forensic Radiology and Imaging (ISFRI) members, and generate a consensus view on a clinical standard protocol.

\section{Materials and methods}

This was a prospective international, multicentre survey of current paediatric postmortem MRI practices and imaging protocols. Institutional ethical review board approval was not required as no patient data were shared or accessed.

An initial fact-finding email (Appendix) was sent to all 17 members of the ISFRI and 14 members of the ESPR Postmortem Imaging Task Force in January 2018 consisting of 5 questions related to paediatric and perinatal postmortem MRI, age ranges and sequences used. Members who did not perform foetal, neonatal or paediatric postmortem MRI were excluded from further correspondence. Two short follow-up surveys were subsequently distributed by email. The lead author (E.W.) developed and designed the two subsequent surveys based in part on the responses to the initial questions.

An initial, 13-question free-text survey (Appendix) was sent to members in March 2018 asking for information regarding the type of MR scanner used, sequence choice, typical duration of scanning and selection of cases.

A second, 10-question multiple-choice survey (Appendix) was sent in January 2019 regarding members' paediatric postmortem MRI experience, training and the impact of their paediatric postmortem MRI service on autopsy referrals. All responses received by March 2019 were included for descriptive analysis.

Survey responses were presented at the Postmortem Imaging Task Force session of the annual ESPR conference in June 2018 in Berlin, Germany [15], and an update was presented at the annual ESPR conference in May 2019 in Helsinki, Finland [16].

Based on the survey results, a recommended clinical standard paediatric postmortem MRI protocol was proposed at the Helsinki meeting and further developed by the task force, with additional imaging considerations adapted from the latest published research on paediatric postmortem MRI.

The final version of this manuscript was circulated among authors in 2020 for consensus approval and formally endorsed by the ESPR board before submission for publication.

\section{Results}

In total, 25 international imaging centres were invited to complete the survey questionnaires. Fourteen responses (56\%) were received. In 2/14 (14\%) centres, paediatric/perinatal postmortem MRI was not performed and 1 of the $14(7 \%)$ centres did not fully complete the questionnaire. Therefore, completed surveys from $11 / 14$ centres $(78 \%)$ were included in our final analysis. Of the completed questionnaires, 13 (93\%) were completed by radiologists who conducted and reported postmortem magnetic resonance imaging examinations and $1(7 \%)$ was completed by a technologist.

The responses originated from the following countries and continents, including:

- Europe: 6/11 (54\%); United Kingdom (2, 18\%), The Netherlands $(2,18 \%)$, Belgium $(1,9 \%)$ and Switzerland (1,9\%).

- Oceania: 4/11 (36\%); Australia (3, 27\%), New Zealand (1, 9\%).

- North America: 1/11 (9\%); Canada (1,9\%).

\section{Referrals and reporting (Table 1)}

All centres performed paediatric postmortem MRI on a scanner located in their main radiology department. None of the centres had a dedicated MRI scanner solely for postmortem imaging, nor one located in the mortuary or pathology department.

Referrers varied between centres but usually included specialists in obstetrics and gynaecology or pathology. Infrequently, a radiologist or parents requested paediatric postmortem MRI. In all centres, the referring doctor communicated the results of the paediatric postmortem MRI to the parents. 


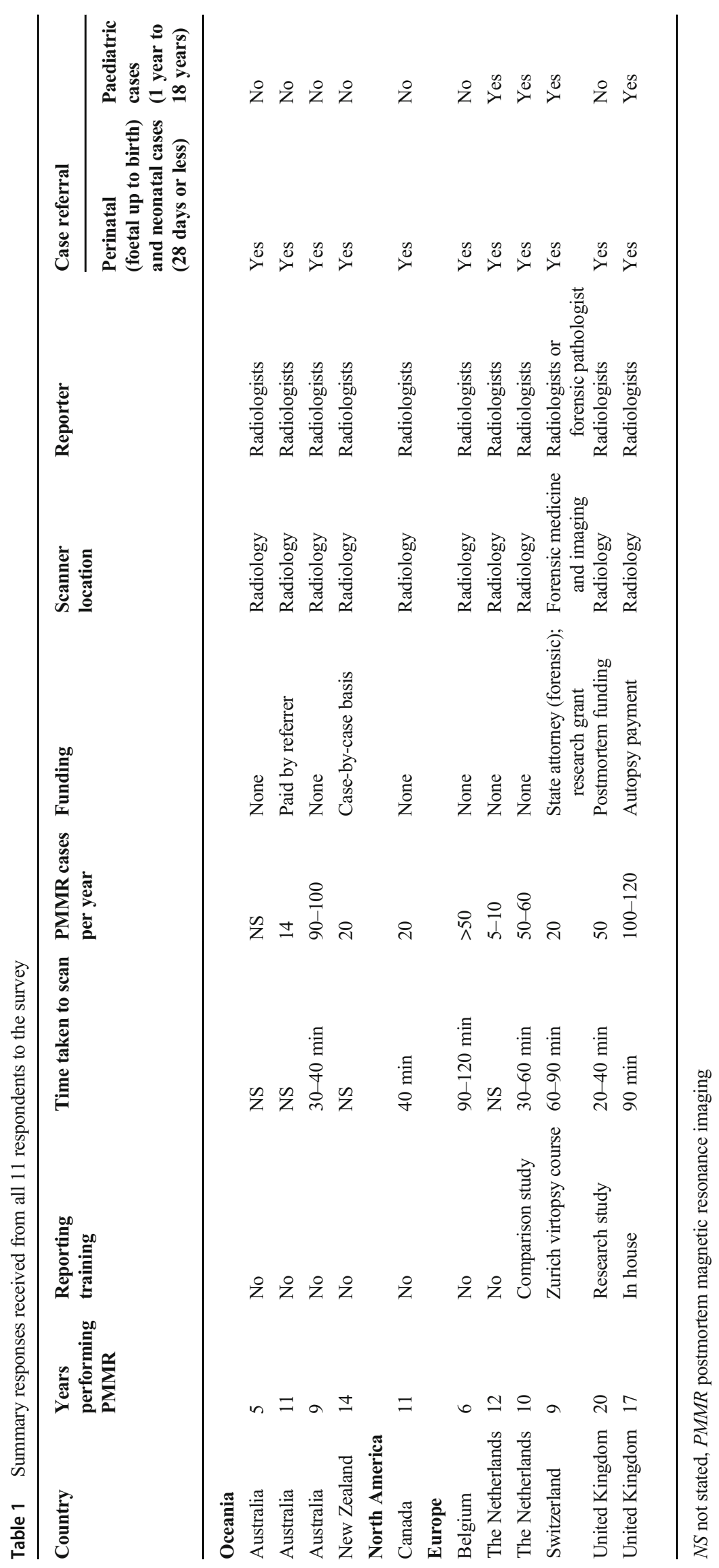


Radiologists were responsible for reporting in all centres, with a forensic pathologist involved for forensic cases at only one centre. Despite this, none of the radiologists had formal training in paediatric postmortem MRI. One $(1 / 11,9.1 \%)$ radiologist had received in-house training from a senior colleague, whilst $2 / 11(18.2 \%)$ centres based their training on research studies undertaken before their clinical services were implemented.

The median duration of paediatric postmortem MRI service provision across all centres was 11 years (range: 5-20 years). The number of cases performed annually ranged from 10 to 120 (mean: 40, median: 20). Most centres believed demand was stable, whereas $4 / 11(36.4 \%)$ centres believed demand was increasing.

All centres provided a paediatric postmortem MRI service for terminations of pregnancy, stillbirths and neonatal deaths. Only 6/11 (54.5\%) centres offered paediatric postmortem MRI services for older children ( $>1$ year). In the Netherlands, this is for the unexpected deaths of children in infancy. Although there is widespread opinion that paediatric postmortem MRI may reduce invasive autopsies, and 4/11 (36.4\%) respondents believed overall conventional autopsy rates in their region were decreasing, none of the respondents had evidence to support this.

None of the centres provide a fully funded, routine service for paediatric postmortem MRI, with the majority performed on a case-by-case basis and the resulting costs absorbed by global hospital or department budgets. Two centres based in the United Kingdom receive some funding as part of the United Kingdom postmortem funding stream (i.e. a portion of the government fee-for-service payment for all paediatric or perinatal conventional autopsies). In the Netherlands, there is government funding for unexpected/unexplained deaths of children from infancy to their 18th birthday.

\section{Imaging protocols (Table 2)}

In only 1/11 (9.1\%) centres, located in Belgium, did the radiologist personally acquire their own MR images. In the other $10 / 11(90.9 \%)$ centres, a radiographer or technologist performed this role. Fourteen scanners were used: 3 (27.2\%) centres used both 3-tesla (T) and 1.5-T scanners, 3 (27.2\%) centres used only a 1.5 -T scanner and $5(45.4 \%)$ centres used only a 3-T scanner. One centre also had access to a 7-T research scanner.

All centres performed T2-weighted sequences in three orthogonal planes, with the addition of at least one T1-weighted sequence, usually in the axial plane for brain and body imaging. Other additional sequences were performed by some institutions, the most frequent being a susceptibility-weighted imaging (SWI) sequence or T2* for detecting haemorrhage $(4 / 11,36.4 \%)$. All radiologists said T2-weighted images were the most useful for interpretation as they provided the most anatomical detail, while diffusion-weighted imaging (DWI) was deemed the least useful.

The average time for a complete paediatric postmortem MRI examination varied between 20 and 120 min (mean: $40 \mathrm{~min}$ ), depending on patient and sequences employed.

The following were among the survey responses:

1) The importance of discussing the findings with the pathologist, if an autopsy was to take place, to ensure that any abnormality seen on imaging was included in histological sections.

2) The importance of taking care when reporting as often there are unexpected findings.

3) The importance of performing postmortem MRI within $24 \mathrm{~h}$ of the request to ensure the parents receive information as quickly as possible.

Table 2 Consensus clinical standard sequences for postmortem foetal brain and body examinations

\begin{tabular}{|c|c|c|c|c|c|c|c|c|}
\hline Sequence & FOV $(\mathbf{m m})$ & $\begin{array}{l}\text { Slice } \\
\text { thickness (mm) }\end{array}$ & Gap & TR (ms) & TE (ms) & $\begin{array}{l}\text { Averages } \\
\text { (NEX/NSA) }\end{array}$ & Voxel size (mm) & $\begin{array}{l}\begin{array}{l}\text { Duration } \\
\text { of sequence } \\
\text { (min) }\end{array} \\
\text { a }\end{array}$ \\
\hline \multicolumn{9}{|l|}{ T2 sequences (brain) } \\
\hline $\begin{array}{l}\text { 2-D T2 TSE } \\
3 \text { planes }\end{array}$ & $180 \times 100$ & 3 & 0.3 & 3,000 & 150 & 2 & $0.8 \times 0.6 \times 3.0$ & 1.56 \\
\hline SWI (if haemorrhage suspected) & $180 \times 148$ & 4 & 0 & 31 & 7.2 & 4 & $0.6 \times 0.6 \times 4.0$ & 1.32 \\
\hline \multicolumn{9}{|l|}{ T2 sequences (body) } \\
\hline $\begin{array}{l}\text { T2 TSE } \\
3 \text { planes }\end{array}$ & $150 \times 119$ & 3 & 0 & 3,870 & 120 & 2 & $0.55 \times 0.59 \times 3.0$ & 3.13 \\
\hline 3-D T2 TSE & $200 \times 200$ & 0.8 & 0 & 3,500 & 275 & 2 & $0.8 \times 0.8 \times 0.8$ & 6.2 \\
\hline \multicolumn{9}{|c|}{ T1 volumetric sequence (brain and body) } \\
\hline T1 3-D & $120 \times 100$ & 2.0 & 0 & 7 & 2.46 & 1 & $1.3 \times 1.3 \times 2.0$ & 1.09 \\
\hline
\end{tabular}

Two-dimensional (2-D) turbo spin echo (TSE) can be used for body images in three planes or a volume sequence

3-D three-dimensional, FOV field of view, NEX number of excitations, NS not stated, NSA number of signal averages, SWI susceptibility-weighted imaging, $T E$ echo time, $T R$ repetition time 
4) The value of allowing technicians/radiographers to choose the timing to fit around their needs, as this reduces the stress of the situation.

5) The value of an international program to help support countries and demonstrate the value of paediatric postmortem MRI to governments and insurance companies.

6) One centre has shown that paediatric postmortem MRI is not as accurate as intrauterine foetal MRI for central nervous system cases [17], a finding supported by another study [18]. Consequently, the centre is now using intrauterine foetal MRI as a first step in postmortem investigations when there is an intention to terminate the pregnancy due to a foetal cranial abnormality.

\section{Discussion}

This prospective survey of a small number of centres performing paediatric postmortem MRI has revealed the common aspects of and variations in referral, technical and reporting practices of paediatric/perinatal postmortem MRI practices internationally and a consensus basic clinical scanning protocol has been developed by the survey participants and task force members. These recommendations will allow for future standardisation of image acquisition and improved ease of multisite reporting.

The recommended imaging sequences are easily adaptable to different patient sizes and scanner manufacturers. Members of the ESPR task force are willing to support other centres in developing paediatric postmortem MRI services and to review imaging scans if requested.
In addition to the standard clinical protocol, specific sequences for brain imaging should include blood-sensitive sequences (e.g., T2* weighted or SWI). Other sequences such as fluid-attenuated inversion recovery (FLAIR) may be included, although their additional diagnostic value in paediatric postmortem MRI is unclear. For body imaging, a threedimensional (3-D) T2-weighted constructive interference steady state (CISS) sequence provides high-resolution cardiac anatomical imaging, although it requires an additional $30 \mathrm{~min}$ of scanning time and may be best reserved for cases where there is a suspected cardiac anomaly [19]. Whole-body short tau inversion recovery (STIR) imaging of the brain may also be helpful, particularly as many radiologists are familiar with this sequence in whole-body live paediatric imaging and believe it provides excellent visualisation of the lamination pattern in the foetal brain. Table 3 details these additional sequences.

The types of coils used were not included in our survey questions, but most centres select the smallest coil the body will fit to maximize signal and obtain as much coverage of the body as possible. Further work is underway to assess the best methods to prepare a body for postmortem imaging, and which imaging modality to use and when in the foetal, neonatal and paediatric age groups.

In all centres, the paediatric postmortem MRI reporting is performed by radiologists, with the majority having no formal training in postmortem imaging. Skills were either self-taught by adapting them from live paediatric or intrauterine foetal imaging experiences or via a postmortem imaging research environment before starting clinical services. There is, therefore, a need for dedicated training programs and guidelines, particularly if more centres are to be encouraged to provide paediatric postmortem imaging services.

Table 3 Additional (optional) sequences for the postmortem MRI examination

\begin{tabular}{|c|c|c|c|c|c|c|c|c|c|}
\hline Sequence & $\begin{array}{l}\text { FOV } \\
(\mathrm{mm})\end{array}$ & Matrix & $\begin{array}{l}\text { Slice } \\
\text { thickness } \\
(\mathbf{m m})\end{array}$ & Gap & $\begin{array}{l}\text { TR } \\
(\mathrm{ms})\end{array}$ & $\begin{array}{l}\text { TE } \\
(\mathrm{ms})\end{array}$ & $\begin{array}{l}\text { Averages } \\
\text { (NEX/ } \\
\text { NSA) }\end{array}$ & $\begin{array}{l}\text { Voxel size } \\
(\mathrm{mm})\end{array}$ & $\begin{array}{l}\text { Duration } \\
\text { (min) }\end{array}$ \\
\hline \multicolumn{10}{|l|}{ Additional 'optional' brain sequences } \\
\hline SWI & $180 \times 148$ & $300 \times 247$ & 4 & 0 & 31 & 7.2 & 4 & $0.6 \times 0.6 \times 4.0$ & 1.32 \\
\hline $\mathrm{T} 2 *$ & $150 \times 122$ & $168 \times 134$ & 3 & 0 & Shortest & 23 & 2 & $0.9 \times 0.9 \times 3.0$ & 3.56 \\
\hline FLAIR (long TR) & $150 \times 117$ & $220 \times 136$ & 3 & 0 & 11,000 & 140 & 3 & $0.75 \times 0.85 \times 3.0$ & 4.46 \\
\hline STIR & $200 \times 200$ & $216 \times 320$ & 4 & 0 & 6,180 & 14 and 115 & 1 & $06 . \times 0.7 \times 4.0$ & 3.19 \\
\hline \multicolumn{10}{|l|}{ Additional 'optional' body sequences } \\
\hline STIR & $200 \times 200$ & $216 \times 320$ & 4 & 0 & 6,180 & 14 and 115 & 1 & $0.6 \times 0.7 \times 4.0$ & 3.19 \\
\hline $\begin{array}{l}\text { 3-D CISS, T2 } \\
\text { (for cardiac pathologies, cover heart } \\
\text { and entire lungs) }\end{array}$ & $150 \times 150$ & $192 \times 256$ & 0.6 & 0 & 5.6 & 2.5 & 10 & $0.6 \times 0.6 \times 0.6$ & 29.26 \\
\hline
\end{tabular}

3-D three-dimensional, CISS constructive interference in a steady state, $D W I$ diffusion-weighted imaging, FLAIR fluid-attenuated inversion recovery, FOV field of view, NEX number of excitations, NSA number of signal averages, STIR short tau inversion recovery, SWI susceptibility-weighted imaging, $T E$ echo time, $T R$ repetition time 
The only published guidelines that we are aware of are the Dutch guidelines for clinical foetal-neonatal and paediatric postmortem radiology [20]. These aim to guide the choice of imaging modality for the size of the body and also take into account the clinical details at presentation.

It is important to note that whilst we included task force members of ESPR and ISFRI, other radiologists, pathologists and medical professionals may perform paediatric postmortem imaging outside these organisations and, therefore, may have been excluded. Nevertheless, this document provides a starting point for consensus guidelines from the current field of expertise.

\section{Conclusion: consensus recommendation}

All centres supported the notion of provision of a consensus clinical standard set of paediatric postmortem MRI sequences that should be included in every examination. This was defined as a basic clinical protocol that:

- Would provide good quality images across all age ranges,

- Is readily achievable in the majority of centres, with minor modifications using any MR scanner model, and

- Allows for multiplanar analysis of key anatomy.

Based on current practices collated from survey responses, the basic clinical standard protocol developed through group consensus included:

- T2-weighted images in three orthogonal planes (axial, coronal, sagittal)

- 3-D T1-weighted isovolumetric sequence

- SWI or T2*-weighted imaging in cases with possible haemorrhage.

The imaging parameters could be adapted to cover the whole body in a single acquisition (brain and torso) or divided into separate brain and torso (thorax and abdomen) acquisitions, largely determined by patient size (Table 2), achievable in under $30 \mathrm{~min}$.

We hope that this will standardise and improve clinical practice, reporting, collaboration and second opinion work, both in clinical and medicolegal practice settings.

Acknowledgments Susan Shelmerdine is supported by a research Council United Kingdom/United Kingdom Research Innovation (RCUK/UKRI) Fellowship and Medical Research Council (MRC) Clinical Research Training Fellowship (Grant Ref: MR/ R002118/1). This award is jointly funded by the Royal College of Radiologists. Owen Arthurs is funded by a National Institute for Health Research (NIHR) Career Development Fellowship (NIHR-CDF-2017-10-037). This article presents independent research and the views expressed are those of the authors and are not necessarily those of the funding bodies.

\section{Compliance with ethical standards}

Conflicts of interest None

\section{Appendix}

\section{Initial fact-finding email}

- Do you currently perform PMMR?

- What age group do you perform PMMR for?

- What sequences do you use?

- How long does your protocol take?

- How many do you perform a month?

Any other information?

\section{Questions in second follow-up email}

- How long have you been performing PMMR for?

- Are you doing PMMR for the brain or whole body or both?

- How long in total does your PMMR protocol take?

- Why have you chosen the sequences you use?

- Which sequences do you find most useful?

- Are there any sequences you do not find useful?

- Do you do 3D volume imaging? Is this to save time? Do you re format in all 3 planes?

- Do you image all cases referred or selected cases? Why?

- Is the number of cases you receive increasing overtime?

- Are you restricted by any local rules?

- Are you restricted by the cost of imaging?

- Are there any aspects you feel are really important to your practice, e.g. certain sequences, reformats, turnaround time, communication of results?

- Do you have anything in your service that you feel is really good practice and would like to see other people use?

\section{ESPR Postmortem Task Force - detailed questionnaire}

1. Do you perform postmortem MRI?

- How long have you been performing PMMR?

- Did you have any training?

- How many do you do an average per year?

- Is this increasing?

- How is this funded in your country?

2. What is the reason you perform PMMR? 
- Is it at parents' request?

- Does the state suggest it?

- Does the coroner or equivalent accept it?

3. Do you know if this has had an impact on the number of full autopsies performed?

- Has this increased or decreased the number of cases that have investigations performed after death?

- Do you still perform autopsy or limited autopsy in cases that have had PMMR?

- What is the overall percentage of cases that have post death investigations in your centre and is this the same, more or less than in your country?

4. Would it be helpful to standardise the sequences used?

- Local standards

- National standards

- International standards

- Have a minimum standard?

5. What is your scanner manufacturer?

6. Where is your scanner located, e.g. radiology department, mortuary, off site?

7. What age group of cases do you scan? Please tick all that apply:

- Foetal

- Terminations of pregnancy (TOP)

- Stillbirths

- Neonates

- All paediatric cases

Any additional details?

8. Who reports your images?

- Radiologist

- Radiographer

- Pathologist

9. Who obtains the images?

- Technicians

- Radiographers

- Radiologists

10. How do the parents obtain the results?

Open Access This article is licensed under a Creative Commons Attribution 4.0 International License, which permits use, sharing, adaptation, distribution and reproduction in any medium or format, as long as you give appropriate credit to the original author(s) and the source, provide a link to the Creative Commons licence, and indicate if changes were made. The images or other third party material in this article are included in the article's Creative Commons licence, unless indicated otherwise in a credit line to the material. If material is not included in the article's Creative Commons licence and your intended use is not permitted by statutory regulation or exceeds the permitted use, you will need to obtain permission directly from the copyright holder. To view a copy of this licence, visit http://creativecommons.org/licenses/by/4.0/.

\section{References}

1. Brookes JA, Hall-Craggs MA, Sams VR, Lees WR (1996) Noninvasive perinatal necropsy by magnetic resonance imaging. Lancet 348:1139-1141

2. Huisman TA, Wisser J, Stallmach T et al (2002) MR autopsy in fetuses. Fetal Diagn Ther 17:58-64

3. Griffiths PD, Variend D, Evans M et al (2003) Postmortem MR imaging of the fetal and stillborn central nervous system. AJNR Am J Neuroradiol 24:22-27

4. Cohen MC, Whitby E (2007) The use of magnetic resonance in the hospital and coronial pediatric postmortem examination. Forensic Sci Med Pathol 3:289-296

5. Cohen MC, Paley MN, Griffiths PD, Whitby EH (2008) Less invasive autopsy: benefits and limitations of the use of magnetic resonance imaging in the perinatal postmortem. Pediatr Dev Pathol 11:1-9

6. Breeze ACG, Gallagher FA, Lomas DJ et al (2008) Postmortem fetal organ volumetry using magnetic resonance imaging and comparison to organ weights at conventional autopsy. Ultrasound Obstet Gynecol 31:187-193

7. Breeze ACG, Jessop FA, Set PAK et al (2011) Minimally-invasive fetal autopsy using magnetic resonance imaging and percutaneous organ biopsies: clinical value and comparison to conventional autopsy. Ultrasound Obstet Gynecol 37:317-323

8. Nicholl RM, Balasubramaniam VP, Urquhart DS et al (2007) Postmortem brain MRI with selective tissue biopsy as an adjunct to autopsy following neonatal encephalopathy. Eur J Paediatr Neurol 11:167-174

9. Thayyil S, Cleary JO, Sebire NJ et al (2009) Post-mortem examination of human fetuses: a comparison of whole-body high-field MRI at 9.4 T with conventional MRI and invasive autopsy. Lancet 374:467-475

10. Votino C, Jani J, Verhoye $\mathrm{M}$ et al (2012) Postmortem examination of human fetal hearts at or below 20 weeks' gestation: a comparison of high-field MRI at 9.4 T with lower-field MRI magnets and stereomicroscopic autopsy. Ultrasound Obstet Gynecol 40:437-444

11. Thayyil S, Sebire NJ, Chitty LS et al (2013) Post-mortem MRI versus conventional autopsy in fetuses and children: a prospective validation study. Lancet 382:223-233

12. Arthurs OJ, Thayyil S, Addison S et al (2014) Diagnostic accuracy of postmortem MRI for musculoskeletal abnormalities in fetuses and children. Prenat Diagn 34:1254-1261

13. Arthurs OJ, Thayyil S, Owens CM et al (2015) Diagnostic accuracy of post mortem MRI for abdominal abnormalities in foetuses and children. Eur J Radiol 84:474-481

14. Arthurs OJ, Thayyil S, Pauliah SS et al (2015) Diagnostic accuracy and limitations of post-mortem MRI for neurological abnormalities in fetuses and children. Clin Radiol 70:872-880

15. Whitby EH (2018) Update on ESPR Paediatric post mortem imaging protocol. Pediatr Radiol 48:S420

16. Whitby EH (2019) Update on PM MR protocols. Pediatr Radiol 49: $312-313$ 
17. Goergen SK, Alibrahim E, Govender N et al (2019) Diagnostic assessment of foetal brain malformations with intra-uterine MRI versus perinatal post-mortem MRI. Neuroradiology 61:921-934

18. Izzo G, Talenti G, Falanga G et al (2019) Intrauterine fetal MR versus postmortem MR imaging after therapeutic termination of pregnancy: evaluation of the concordance in the detection of brain abnormalities at early gestational stage. Eur Radiol 29:2740-2750

19. Taylor AM, Sebire NJ, Ashworth MT et al (2014) Postmortem cardiovascular magnetic resonance imaging in fetuses and children: a masked comparison study with conventional autopsy. Circulation 129:1937-1944

20. Sonnemans LJP, Vester MEM, Kolsteren EEM et al (2018) Dutch guideline for clinical foetal-neonatal and paediatric post-mortem radiology, including a review of literature. Eur J Pediatr 177:791803

Publisher's note Springer Nature remains neutral with regard to jurisdictional claims in published maps and institutional affiliations. 